\title{
VERBAL VARIETIES OF INVERSE SEMIGROUPS
}

\author{
ROSS WILKINSON
}

(Received 19 October 1982)

Communicated by T. E. Hall

\begin{abstract}
A verbal product is introduced for a particular class of varieties of inverse semigroups and this product is shown to be associative. As well, the structure of this class is examined.
\end{abstract}

1980 Mathematics subject classification (Amer. Math. Soc.): 20 M 05.

\section{Introduction}

In 1937, B. H. Neumann [11] introduced the concept of a verbal subgroup. If $G$ is a group and $w$ is a set of words in $F G_{X}$, the free group on a set $X$, then the verbal subgroup $w(G)$ of $G$ generated by $w$ is the set consisting of all elements $g$ of $G$ for which there is a morphism from $F G_{X}$ to $G$ mapping an element of $w$ to $g$. Such subgroups are fully invariant (and hence normal) but the converse is not the case.

S. Moran [10], in an examination of associative products of groups, introduced a verbal product of groups, which is associative and as well is commutative. If $G$ and $H$ are groups, and $G \pi H$ denotes their free product and $[G, H]$ denotes all commutators of the form $[g, h]$ or $[h, g]$ where $g \in G$ and $h \in H$, then the w-verbal product is $(G \pi H) / \mathrm{w}(G \pi H) \cap[G, H]$.

We may take another approach and fix the group and vary the verbal subgroup and obtain another associative product. This was the approach of $H$. Neumann [12]. The fixed group is $F G_{X}$ and if $u$ and $v$ are verbal subgroups of $F G_{X}$, then the product she defines is

$$
F G_{X} / \mathbf{u} \circ F G_{X} / \mathbf{v}=F G_{X} / \mathbf{u}(\mathbf{v})
$$

(C) 1984 Australian Mathematical Society $0263-6115 / 84 \$ A 2.00+0.00$ 
This product is associative since $(\mathbf{u}(\mathbf{v}))(\mathbf{w})=\mathbf{u}(\mathbf{v}(\mathbf{w}))$. Thus $\mathrm{H}$. Neumann was able to define an associative product of varieties of groups with the product of two varieties being given by the relatively free group defined above.

A striking result established by $\mathrm{H}$. Neumann was that this product of varieties is equivalent to another product determined by extensions of groups of the first variety by groups of the second variety. The aim of this paper is to define a verbal product for a certain class of varieties of inverse semigroups. We see that this product is associative. As well we examine the structure of this class of varieties and generalise the above result to semilattices of groups.

\section{Verbal varieties}

Let $X$ be a fixed countably infinite set, $X=\left\{x_{1}, x_{2}, x_{3}, \ldots\right\}$, and $F I_{X}$ be the free inverse semigroup on $X$. An element of $F I_{X}$ is called a word. A pair of words $(u, v)$ is called an identity of an inverse semigroup, $S$, if $u \alpha=v \alpha$ for all morphisms $\alpha: F I_{X} \rightarrow S$, and we say $S$ satisfies $(u, v)$. We shall call a word $w$ and idempotent law of $S$ if for all morphisms $\alpha: F I_{X} \rightarrow S, w \alpha \in E(S)$, the set of idempotents of $S$, and we say $S$ satisfies $w$. Notice $w$ is an idempotent law of $S$ if and only if $\left(w, w w^{-1}\right)$ is an identity of $S$.

DEFINITION 1. For an inverse semigroup, $S$, the verbal inverse subsemigroup of $S$, denoted by $\mathbf{w}(S)$, corresponding to a set of words $\mathbf{w}$ is the inverse subsemigroup of $S$ generated by $\left\{w \alpha \mid w \in w, \alpha \in \operatorname{Mor}\left(F I_{X}, S\right)\right\}$.

A variety of inverse semigroups is a collection of inverse semigroups closed under the operation of taking inverse subsemigroups, forming morphic images and forming direct products. Naturally, we say that a variety of inverse semigroups satisfies $(u, v)$ if each inverse semigroup in the variety satisfies $(u, v)$ and a variety of inverse semigroups satisfies a word $w$ if each inverse semigroup in the variety satisfies $w$.

As a special case of a result of Birkhoff [2] we know that $\mathfrak{V}$ is a variety of inverse semigroups if and only if there is a set $A \subseteq F I_{X} \times F I_{X}$ such that $\mathfrak{W}$ is the collection of all inverse semigroups satisfying each pair $(u, v)$ in $A$. We shall say that $\mathfrak{V}$ is the variety defined by $A$, and denote it by $\mathscr{V}_{A}$.

DEFINITION 2. A variety of inverse semigroups that is defined by a set $A=\left\{\left(w, w w^{-1}\right) \mid w \in \mathbf{w} \subseteq F I_{X}\right\}$ is called a verbal variety, and is denoted by $\mathcal{V}_{\mathbf{w}}$.

Notice that $\mathfrak{V}_{w}=\mathfrak{V}_{A}$. It follows that $\mathfrak{V}_{w}$ consists of all inverse semigroups such that each $w \in \mathbf{w}$ is an idempotent law. 
Definition 3. If $w$ is a set of words, the set of all idempotent laws satisfied by $\mathfrak{V}_{\mathbf{w}}$ is called the total set generated by $\mathbf{w}$, and is denoted by $T(\mathbf{w})$.

We shall investigate the structure of the lattice of verbal varieties a little later in Section 5 but first we make some brief observations.

Let $\mathbf{w} \subseteq E\left(F I_{X}\right)$, the semilattice of idempotents of the free inverse semigroup on a set $X$; then every inverse semigroup satisfies each of the idempotent laws $w \in \mathbf{w}$, since the morphic image of an idempotent is necessarily an idempotent. Thus the variety of all inverse semigroups, denoted $9 \mathcal{S}$, is a verbal variety.

Now if $x \in X$, then $S$ satisfies $x$ only if $S$ is a semilattice since otherwise if $s^{2} \neq s \in S$ then the morphism generated by $x_{i} \rightarrow s, x_{i} \in X$ shows that $S$ does not satisfy $x$. Thus if $\mathbf{w}=F I_{X}$, then only a semilattice can satisfy all $w \in \mathbf{w}$. Certainly, however, every semilattice satisfies every word in $F I_{X}$, so the variety of semilattices, denoted $\mathcal{E} \mathcal{E}$, is the smallest verbal variety.

The variety, $\mathcal{L} \mathcal{L}$, is not the only verbal variety to have trivial intersection with the variety of groups $\mathcal{G}$. If $\mathcal{C}_{n}, n \geqslant 1$, denotes the variety of inverse semigroups defined by the identity $\left(x^{n+1}, x^{n}\right)$, then each $\mathcal{C}_{n}$ is an $\mathcal{G}$-degenerate variety, that is each inverse semigroup in the variety has each of its $\mathcal{G}$-classes containing only one element. Thus each $\bigodot_{n}$ has only one element groups in it. Each $\bigodot_{n}$ is a verbal variety, since $\left(x^{n+1}, x^{n}\right)$ and $\left(\left(x^{n} x^{1-n}\right)\left(x^{n} x^{1-n}\right)^{-1}, x^{n} x^{1-n}\right)$ are equivalent identities. These varieties are of some significance, since Djadchenko [4] shows that every $\mathcal{G}$-degenerate variety of inverse semigroups satisfies $\left(x^{n+1}, x^{n}\right)$ for some $n \geqslant 1$.

We shall see later that not every variety of inverse semigroups is a verbal variety. There are however, "plenty" of verbal varieties. Given any variety of inverse semigroups, $\mathcal{T}$, we may obtain the smallest verbal variety containing it by taking the variety of inverse semigroups satisfying $\left(w w^{-1}, w\right)$ for all identities of this form satisfied by $\mathcal{V}$. In the lattice of varieties of inverse semigroups, this is a closure operation and so we shall denote the verbal variety obtained in this way from a variety $\mathfrak{T}$, by $\mathfrak{V}^{c}$.

\section{Fully invariant congruences and normal inverse subsemigroups}

We call a congruence $\rho$ on an algebra $A$ fully invariant if for all endomorphisms $\phi: A \rightarrow A$ and for all $x, y \in A$, we have $(x, y) \in \rho$ implies $(x \phi, y \phi) \in \rho$. Further, an inverse subsemigroup, $A$ of an inverse semigroup $S$ is said to be fully invariant in $S$, if for each endomorphism $\phi$ of $S, A \phi \subseteq A$.

Fully invariant congruences, introduced by Birkhoff [2], play a central role in studying varieties and the key result when specialized to inverse semigroups, gives 
LEMMA 4. Let $R$ be a set of pairs of words in $F I_{X}$. Then $\mathfrak{V}_{R}$, the variety defined by $R$, satisfies all identities $(u, v)$, where $(u, v) \in \rho_{R}^{*}$, the fully invariant congruence of $F I_{X}$ generated by $R$, and only those identities.

The construction of a fully invariant congruence from a relation $R$ may be achieved as follows.

LeMMA 5. Let $R$ be a relation on a semigroup $S$. Let $R_{0}=R$ and let $R_{i}=\left\{(a, b) \in S \times S \mid(a, b)=(c \theta, d \theta)\right.$ with $(c, d) \in \rho_{i-1}$ and $\left.\theta \in \operatorname{End}(S)\right\}$ where $\rho_{i-1}$ is the congruence on $S$ generated by $R_{i-1}, i \geqslant 1$. Let $\rho_{R}^{*}=\cup_{i=0}^{\infty} R_{i}$ (and hence $\left.=\cup_{i=0}^{\infty} \rho_{i}\right)$. Then $\rho_{R}^{*}$ is the fully invariant congruence on $S$, generated by $R$. (See Clifford and Preston [3] page 18 for a constructive definition of $\rho_{i}$.)

COROLLARY. Let $R$ be a relation on an inverse semigroup $S$. Then $\rho_{R}^{*}$, as defined in the lemma, is the fully invariant congruence on $S$ generated by $R$.

Definition 6. Let $\phi: S \rightarrow T$ be a morphism of inverse semigroups. The core of $\phi$, written core $\phi$, is $\left\{x \in S \mid x \phi=\left(x x^{-1}\right) \phi\right\}$.

We recall the following definition by Green [5].

DEFINITION 7. Let $N$ be an inverse subsemigroup of an inverse semigroup $S$. Then $N$ is normal in $S$ (or where it is clear simply normal) if

(i) $E(S) \subseteq N$, that is $N$ is full, and

(ii) for all $x, y \in S$ and for all $n \in N, x y \in N$ implies $x n y \in N$.

DEFINITION 8. If $S$ is an inverse semigroup and $N$ is an inverse subsemigroup of $S$, then we denote by $\rho_{N}$, the congruence on $S$ generated by $\left\{\left(n, n n^{-1}\right) \mid n \in N\right\}$.

We slightly extend a result of Green [5] to consider the case of full invariance, to obtain the following most useful result, that perhaps justifies the use of the term "normal".

LEMMA 9. For any $\phi: S \rightarrow T$, a morphism of inverse semigroups, core $\phi$ is normal. Conversely, for any normal inverse subsemigroup $N$ of an inverse semigroup of $S$, there is a congruence on $S$, namely $\rho_{N}$, with $\operatorname{core} \rho_{N}=N$. Further $N$ is fully invariant if and only if $\rho_{N}$ is fully invariant.

Proof. It is clear that core $\phi$ is a full inverse subsemigroup of $S$. Normality follows essentially because, for any inverse semigroup, and hence for $T$, if $e$ and 
$a b$ are each idempotent, then so is $a e b$. To show the converse, we first consider the relation $\tau$ where $(a, b) \in \tau$ if and only if there exist $r_{1}, \ldots, r_{n}, s_{1}, \ldots, s_{n} \in S^{1}$ and $w_{1}, \ldots, w_{n} \in N$ such that

$$
\begin{array}{ccc}
a=r_{1} w_{1} s_{1} & c_{1}=r_{1} w_{1} w_{1}^{-1} s_{1} \\
c_{1}=r_{2} w_{2} s_{2} & c_{2}=r_{2} w_{2} w_{2}^{-1} s_{2} \\
& \vdots & \\
c_{n-1}=r_{n} w_{n} s_{n} & b=r_{n} w_{n} w_{n}^{-1} s_{n}
\end{array}
$$

Now $\left\{\left(n, n n^{-1}\right) \mid n \in N\right\} \subseteq \tau$ and also by the definition of $\tau, \tau \subseteq \rho_{N}$. But we shall see that $\tau$ is a congruence; so $\tau=\rho_{N}$. Since $a=a\left(a^{-1} a\right) 1$ and $a=$ $a\left(a^{-1} a\right)\left(a^{-1} a\right)^{-1} 1,(a, a) \in \tau$ for any $a \in S$. Let $(a, b) \in \tau$ with (1) holding. Thus

$$
\begin{array}{ccc}
b=\left(r_{n} w_{n}\right) w_{n}^{-1} s_{n} & c_{n-1}=\left(r_{n} w_{n}\right) w_{n}^{-1} w_{n} s_{n} \\
c_{1}=\left(r_{1} w_{1}\right) w_{1}^{-1} s_{1} & a=\left(r_{1} w_{1}\right) w_{1}^{-1} w_{1} s_{1}
\end{array}
$$

so that $(b, a) \in \tau$. It is clear that $\tau$ is transitive. Let $(a, b) \in \tau$ and let (1) hold. Let $x, y \in S^{1}$, so

$$
\begin{array}{cc}
x a y=\left(x r_{1}\right) w_{1}\left(x_{1} y\right) \quad & x c_{1} y=\left(x r_{1}\right) w_{1} w_{1}^{-1}\left(s_{1} y\right) \\
\vdots & \\
& x b y=\left(x r_{n}\right) w_{n} w_{n}^{-1}\left(s_{n} y\right)
\end{array}
$$

and so $(x a y, x b y) \in \tau$. Thus $\tau$ is a congruence and $\tau=\rho_{N}$.

Now clearly $N \subseteq$ core $\rho_{N}$. Let $a \in \operatorname{core} \rho_{N}$. Thus $\left(a, a a^{-1}\right) \in \tau$ so equalities of the following form hold:

$$
\begin{array}{cc}
a=r_{1} w_{1} s_{1} & c_{1}=r_{1} w_{1} w_{1}^{-1} s_{1} \\
& \vdots \\
c_{n-1}=r_{n} w_{n} s_{n} & a a^{-1}=r_{n} w_{n} w_{n}^{-1} s_{n}
\end{array}
$$

Now $a a^{-1} \in N$ and $w_{n} \in N$ so by condition (ii) defining normality, $c_{n-1}=$ $\left(r_{n} w_{n} w_{n}^{-1}\right) w_{n}\left(s_{n}\right) \in N$. But $c_{n-1} \in N$ and $w_{n-1} \in N$ so, as for $c_{n-1}, c_{n-2}=$ $\left(r_{n-1} w_{n-1} w_{n-1}^{-1}\right) w_{n-1}\left(s_{n-1}\right) \in N$, and so on. Thus we find that $c_{n-1}, \ldots, c_{1} \in N$, and finally that $a \in N$. Hence $N=\operatorname{core} \rho_{N}$.

Finally, suppose that $N$ is fully invariant and that $(a, b) \in \rho_{N}$. Then for all endomorphisms $\theta: S \rightarrow S$,

$$
a \theta=\left(r_{1} w_{1} s_{1}\right) \theta \quad \begin{gathered}
c_{1} \theta=\left(r_{1} w_{1} w_{1}^{-1} s_{1}\right) \theta \\
\vdots \quad \\
b \theta=\left(r_{n} w_{n} w_{n}^{-1} s_{n}\right) \theta
\end{gathered}
$$


But for all $i,\left(r_{i} w_{i} s_{i}\right) \theta=\left(r_{i} \theta\right)\left(w_{i} \theta\right)\left(s_{i} \theta\right)$, and as $N$ is fully invariant $w_{i} \theta \in N$, and hence $(a \theta, b \theta) \in \rho_{N}$. Conversely if $\rho_{N}$ is fully invariant and $a \in N=\operatorname{core} \rho_{N}$, then $\left(a, a a^{-1}\right) \in \rho_{N}$ so $\left(a \theta,\left(a a^{-1}\right) \theta\right) \in \rho_{N}$ and hence $\left(a \theta, a \theta(a \theta)^{-1}\right) \in \rho_{N}$ since $\theta$ is morphic. Thus $a \theta \in \operatorname{core} \rho_{N}$ so $a \theta \in N$. Thus $N=\operatorname{core} \rho_{N}$ is fully invariant.

The proof given here is different from that of Green. When the congruence is constructed as in this proof then it becomes easy to see that if $N$ is fully invariant, then so is $\rho_{N}$. This fact is not obvious otherwise. Notice that unlike the situation for groups, we need to show normality as well as full invariance. The following example illustrates this.

EXAMPLE 10. Let $S$ be the inverse semigroup of one to one mappings generated by $\left(\begin{array}{ll}1 & 2 \\ 2 & 3\end{array}\right)$. Then

$$
\begin{array}{r}
S=\left\{\varnothing,\left(\begin{array}{l}
1 \\
1
\end{array}\right),\left(\begin{array}{l}
2 \\
2
\end{array}\right),\left(\begin{array}{l}
3 \\
3
\end{array}\right),\left(\begin{array}{l}
1 \\
2
\end{array}\right),\left(\begin{array}{l}
2 \\
1
\end{array}\right),\left(\begin{array}{l}
1 \\
3
\end{array}\right),\left(\begin{array}{l}
3 \\
1
\end{array}\right),\left(\begin{array}{l}
2 \\
3
\end{array}\right),\left(\begin{array}{l}
3 \\
2
\end{array}\right),\right. \\
\left.\left(\begin{array}{ll}
1 & 2 \\
1 & 2
\end{array}\right),\left(\begin{array}{ll}
2 & 3 \\
2 & 3
\end{array}\right),\left(\begin{array}{ll}
1 & 2 \\
2 & 3
\end{array}\right),\left(\begin{array}{ll}
2 & 3 \\
1 & 2
\end{array}\right)\right\} .
\end{array}
$$

Let $N$ be the inverse subsemigroup

$$
\left\{\varnothing,\left(\begin{array}{l}
1 \\
1
\end{array}\right),\left(\begin{array}{l}
2 \\
2
\end{array}\right),\left(\begin{array}{l}
3 \\
3
\end{array}\right),\left(\begin{array}{l}
1 \\
3
\end{array}\right),\left(\begin{array}{l}
3 \\
1
\end{array}\right),\left(\begin{array}{ll}
1 & 2 \\
1 & 2
\end{array}\right),\left(\begin{array}{ll}
2 & 3 \\
2 & 3
\end{array}\right)\right\} .
$$

Now if $N$ is not fully invariant, then there exists $\theta \in \operatorname{End}(S)$ and $a \in S \backslash N$ such that $\left(\begin{array}{l}1 \\ 3\end{array}\right) \theta=a$. Now suppose $\left(\begin{array}{ll}2 & 3 \\ 1 & 2\end{array}\right) \theta=b$. Then

$$
a a^{-1}=\left(\begin{array}{l}
1 \\
3
\end{array}\right) \theta\left(\begin{array}{l}
3 \\
1
\end{array}\right) \theta=\left(\begin{array}{l}
1 \\
1
\end{array}\right) \theta=\left[\left(\begin{array}{l}
1 \\
3
\end{array}\right)\left(\begin{array}{ll}
2 & 3 \\
1 & 2
\end{array}\right)\left(\begin{array}{ll}
2 & 3 \\
1 & 2
\end{array}\right)\right] \theta=a b^{2} .
$$

However a check of the multiplication table of $S$ reveals that there exists $a b$ such that $a a^{-1}=a b^{2}$ only if $a \in N$. Thus $N$ is fully invariant. However $N$ is not normal since $\left(\begin{array}{ll}1 & 2 \\ 2 & 3\end{array}\right)\left(\begin{array}{l}1 \\ 3\end{array}\right)=\varnothing \in N$ and $\left(\begin{array}{l}3 \\ 1\end{array}\right) \in N$ but $\left(\begin{array}{ll}1 & 2 \\ 2 & 3\end{array}\right)\left(\begin{array}{l}3 \\ 1\end{array}\right)\left(\begin{array}{l}1 \\ 3\end{array}\right)=\left(\begin{array}{l}2 \\ 3\end{array}\right) \notin N$.

We now give a lemma needed later.

LEMMA 11. Every total set of words is a fully invariant normal inverse subsemigroup of $F I_{X}$.

Proor. Let $w$ be a total set of words, then by Lemma $4, \rho_{w}^{*}$ is all the identities satisfied in $\widetilde{V}_{w}$. Thus $w=\left\{w \mid\left(w, w w^{-1}\right)\right.$ is an identity satisfied in $\left.\mathcal{V}_{w}\right\}=$ core $\rho_{w}^{*}$. Now $\rho_{w}^{*}$ is fully invariant, so by Lemma $9, w$ is a fully invariant normal inverse subsemigroup of $F I_{X}$.

COROLlaRY. If $\mathbf{w}=T(\mathbf{w}), \mathbf{w}\left(F I_{X}\right)=\mathbf{w}$. 
Proof. Since $\mathbf{w}$ is fully invariant in $F I_{X}, w \alpha \in \mathbf{w}$, for any $w \in \mathbf{w}$ and $\alpha \in$ $\operatorname{End}\left(F I_{X}\right)$. Thus $\mathbf{w}=\mathbf{w}\left(F I_{X}\right)$.

\section{The verbal product of verbal varieties}

Before introducing the verbal product we need a little more notation. If $u$ is a word in $F I_{X}$, let $c(u)$ denote the content of $u$, that is the set of elements explicitly used in the word $u$. Thus $c\left(x_{1} x_{2}^{2} x_{1}^{-1}\right)=\left\{x_{1}, x_{2}\right\}$ and $c\left(x_{1}^{2} x_{1}^{-1} x_{3} x_{4}\right)=\left\{x_{1}, x_{3}, x_{4}\right\}$. Further if $c(u)=\left\{x_{1}, x_{2}, \ldots, x_{n}\right\}$ then we may write $u=u\left(x_{1}, x_{2}, \ldots, x_{n}\right)$.

If $w_{1}, w_{2}, \ldots, w_{n} \in F I_{X}$ and $c(u)=\left\{x_{1}, \ldots, x_{n}\right\}$, then we may write $u\left(w_{1}, w_{2}, \ldots, w_{n}\right)$ for the word formed by replacing each instance of $x_{i}$ in $u$ by the word $w_{i}$, for $i=1, \ldots, n$.

LEMMA 12. Let $\mathrm{w}$ be a total set of words in $\mathrm{FI}_{X}$. Let $S$ be an inverse semigroup. Then the verbal inverse subsemigroup $\mathrm{w}(S)=\left\{w \alpha \mid w \in \mathrm{w}\right.$ and $\left.\alpha \in \operatorname{Mor}\left(F I_{X}, S\right)\right\}$ and furthermore $\mathrm{w}(S)$ is fully invariant.

Proof. $w(S)=\left\{w \alpha \mid w \in w\right.$ and $\left.\alpha \in \operatorname{Mor}\left(F I_{X}, S\right)\right\}=T$, say, if and only if $T$ is already an inverse subsemigroup of $S$. Let $x=u \alpha$ and $y=v \beta$, where $u, v \in \mathbf{w}$ and $\alpha, \beta \in \operatorname{Mor}\left(F I_{X}, S\right)$. Now choose $\phi \in \operatorname{End}\left(F I_{X}\right)$ such that $c(u) \cap c(v \phi)=$ $\varnothing$. Then $u, v \phi \in \mathrm{w}$ and there exists $\gamma: F I_{X} \rightarrow S$ with $u \alpha=u \gamma$ and $(v \phi) \gamma=v \beta$, whence $x y=(u \alpha)(v \beta)=u \gamma(v \phi) \gamma=(u(v \phi)) \gamma \in T$, all since $w$ is a fully invariant inverse subsemigroup of $F I_{X}$, by Lemma 11. Also, again by Lemma 11 , since $w \in \mathbf{w}$ implies $w^{-1} \in \mathbf{w}, w \alpha \in T$ implies $(w \alpha)^{-1} \in T$, so $T$ is an inverse subsemigroup, and hence $\mathrm{w}(S)=T$. Finally, if $u \alpha \in T$ and $\theta \in \operatorname{End}(S)$, then $\alpha \theta \in \operatorname{Mor}\left(F I_{X}, S\right)$ so $(u \alpha) \theta=u(\alpha \theta) \in T$, so $\mathbf{w}(S)$ is fully invariant.

It is not the case, however, that a verbal inverse semigroup of a total set is necessarily normal, since if $w=T\left(x^{2}\right)$, for some $x \in X$, and $S$ is the inverse semigroup generated by $\left(\begin{array}{ll}1 & 2 \\ 2 & 3\end{array}\right)$, the inverse semigroup mentioned in the previous example, then

$$
N=\left\{\varnothing,\left(\begin{array}{l}
1 \\
1
\end{array}\right),\left(\begin{array}{l}
2 \\
2
\end{array}\right),\left(\begin{array}{l}
3 \\
3
\end{array}\right),\left(\begin{array}{l}
1 \\
3
\end{array}\right),\left(\begin{array}{l}
3 \\
1
\end{array}\right),\left(\begin{array}{ll}
1 & 2 \\
1 & 2
\end{array}\right),\left(\begin{array}{ll}
2 & 3 \\
2 & 3
\end{array}\right)\right\}=w(S)
$$

which was shown not to be normal in $S$.

We note in passing that the operation of obtaining a total set of words from a set of words in $F I_{X}$ is a closure operation on the lattice of subsets of $F I_{X}$. It is also clear from the definition of a total set that $\mathfrak{V}_{\mathbf{u}}=\mathfrak{V}_{T(\mathbf{u})}$, and further that if $\mathbf{u}$ and $\mathbf{v}$ are subsets of $F I_{X}$, since $T(\mathrm{v})$ is an inverse subsemigroup of $F I_{X}$, by Lemma 11, we may form $T(\mathrm{u})(T(\mathrm{v}))$, the verbal inverse subsemigroup of $T(\mathrm{v})$. 
Definirion 13. Let $\mathcal{V}_{u}$ and $\mathcal{V}_{v}$ be the verbal varieties defined by the sets of idempotent laws $\mathbf{u}$ and $\mathbf{v}$, respectively. Let $\mathfrak{V}_{\mathbf{u}} * \mathscr{V}_{\mathbf{v}}=\mathscr{V}_{T(\mathbf{u})(T(v))}$, where $T(\mathbf{u})(T(\mathbf{v}))$ is the verbal inverse subsemigroup of $T(v)$ for the set of words $T(u) . \mathscr{T}_{u} * \mathscr{V}_{v}$ will be called the verbal product of the varieties $\mathfrak{V}_{u}$ and $\mathfrak{V}_{v}$.

THEOREM 14. The verbal product of verbal varieties of inverse semigroups is associative.

Proof. Let $\mathscr{V}_{u}, \mathcal{V}_{v}$ and $\mathfrak{V}_{\mathbf{w}}$ be verbal varieties defined by $u, v$ and $\mathbf{w}$ respectively. Since the product is defined in terms of total sets and since $\mathfrak{V}_{u}=\mathscr{V}_{T(u)}$, we may and shall assume that $\mathbf{u}, \mathbf{v}$ and $\mathbf{w}$ are total. Thus, all that is required is that we show that $[\mathbf{u}(\mathbf{v})] \mathbf{w}=\mathbf{u}(\mathbf{v}(\mathbf{w}))$. Let $z \in[\mathbf{u}(\mathbf{v})] \mathbf{w}$. Then there exist morphisms $\theta$ : $F I_{X} \rightarrow \mathrm{v}$ and $\chi: F I_{X} \rightarrow \mathrm{w}$ and $u \in \mathrm{u}$ such that $(u \theta) \chi=z$. However $\theta \chi: F I_{X} \rightarrow \mathrm{v}(\mathbf{w})$ so that $z=(u \theta) \chi=u(\theta \chi) \in \mathbf{u}(\mathbf{v}(\mathbf{w}))$. Thus $[\mathbf{u}(\mathbf{v})] \mathbf{w} \subseteq \mathbf{u}(\mathbf{v}(\mathbf{w}))$.

Conversely, suppose $z \in \mathrm{u}(\mathrm{v}(\mathrm{w}))$. Then there exist a morphism $\alpha: F I_{X} \rightarrow \mathrm{v}(\mathbf{w})$ and $u \in \mathrm{u}$ such that $u \alpha=z$. Now if $P=\{1,2,3, \ldots\}, \alpha$ is defined by $x_{i} \rightarrow v_{i} \theta_{i}$, for each $i \in P$, where each $v_{i} \in \mathrm{v}$ and each $\theta_{i} \in \operatorname{Mor}\left(F I_{X}, \mathbf{w}\right)$. Now since the naming of the elements of $X$ was arbitrary we may assume that $u=u\left(x_{1}, \ldots, x_{n}\right)$, that is $c(u)=\left\{x_{1}, \ldots, x_{n}\right\}$. Let $x_{r}$ be the element of $X$ in $\bigcup_{i=1}^{n} c\left(v_{i}\right)$ with largest subscript. Suppose that for $1 \leqslant i \leqslant n, a_{i}: P \rightarrow P$ is chosen such that $v_{i}=$ $v_{i}\left(x_{a_{i}(1)}, \ldots, x_{a_{i}(m(i))}\right)$, where $m(i)=\left|c\left(v_{i}\right)\right|$. Now define $\beta: F I_{X} \rightarrow \mathrm{v}$ by

$$
\begin{aligned}
& x_{1} \beta=v_{1}\left(x_{a_{1}(1)}, \ldots, x_{a_{1}(m(1))}\right), \\
& x_{2} \beta=v_{2}\left(x_{r+a_{2}(1)}, \ldots, x_{r+a_{2}(m(2))}\right), \\
& \vdots \\
& x_{n} \beta=v_{n}\left(x_{(n-1) r+a_{n}(1)}, \ldots, x_{\left.(n-1) r+a_{n}(m(n))\right)}\right), \\
& x_{n+s} \beta=v,
\end{aligned}
$$

where $v$ is an arbitrary element of $\mathrm{v}$ and $s$ is any element of $P . \beta$ is a morphism into $\mathrm{v}$ since $\mathrm{v}$ is fully invariant and hence if $v_{i}\left(x_{a_{i}(1)}, \ldots, x_{a_{i}(m(i))}\right)$ is in $\mathrm{v}$ then so is $v_{i}\left(x_{(i-1) r+a_{i}(1)}, \ldots, x_{(i-1) r+a_{i}(m(i))}\right)$. Now define a morphism $\gamma: F I_{X} \rightarrow \mathrm{w}$ by,

$$
\gamma: \begin{cases}x_{(i-1) r+a_{i}(j)} \rightarrow\left(x_{a_{i}(j)}\right) \theta_{i}, & 1 \leqslant j \leqslant m(i), \\ x_{k} \rightarrow w, & \text { if } k \neq(i-1) r+a_{i}(j),\end{cases}
$$

for any $i=1, \ldots, n$ and $j=1, \ldots, m(i)$, and some arbitrary choice of $w$. Notice that $\gamma$ is well defined due to the choice of $r$. Also $\left(F I_{X}\right) \gamma \subseteq$ w since each $\theta_{i}$ : 
$F I_{X} \rightarrow$ w. Also for any $i=1, \ldots, n$,

$$
\begin{aligned}
x_{i} \beta \gamma & =v_{i}\left(x_{(i-1) r+a_{i}(1)}, \ldots, x_{(i-1) r+a_{i}(m(i))}\right) \gamma \\
& =v_{i}\left(x_{(i-1) r+a_{i}(1)} \gamma, \ldots, x_{(i-1) r+a_{i}(m(i))} \gamma\right) \\
& =v_{i}\left(x_{a_{i}(1)} \theta_{i}, \ldots, x_{a_{i}(m(i))} \theta_{i}\right) \\
& =v_{i}\left(x_{a_{i}(1)}, \ldots, x_{a_{i}(m(i))}\right) \theta_{i} \\
& =v_{i} \theta_{i} \\
& =x_{i} \alpha .
\end{aligned}
$$

Thus $u \beta \gamma=u \alpha=z$, whence $z \in[u(v)] \mathbf{w}$, so $\mathbf{u}(\mathbf{v}(\mathbf{w})) \subseteq[\mathbf{u}(\mathbf{v})] \mathbf{w}$. The result of the theorem follows.

LEMMA 15. Let $\mathfrak{T}_{u}, \mathfrak{V}_{v}$ be verbal varieties of inverse semigroups. Let $S$ be an inverse semigroup such that there exists a congruence, $\rho$, on $S$ with core $\rho \in \mathscr{V}_{\mathrm{u}}$ and $S / \rho \in \mathfrak{V}_{v}$. Then $S \in \mathfrak{V}_{u} * \mathfrak{T}_{v}$.

Proof. Let $u\left(v_{1}, \ldots, v_{n}\right) \in T(\mathbf{u})(T(\mathbf{v}))$, where $u\left(x_{1}, \ldots, x_{n}\right) \in T(\mathbf{u})$ and $v_{i} \in T(\mathbf{v})$ for $i=1, \ldots, n$. Let $\theta \in \operatorname{Mor}\left(F I_{X}, S\right)$. Now $\left(v_{i} \theta\right) \rho \in E(S / \rho)$ for each $v_{i}$, as $S / \rho \in V_{\mathrm{v}}$ and $\theta \rho: F I_{X} \rightarrow S / \rho$, so $v_{i} \theta \in \operatorname{core} \rho^{\natural}=\operatorname{core} \rho$. Thus $\left(u\left(v_{1}, \ldots, v_{n}\right)\right) \theta=$ $u\left(v_{1} \theta, \ldots, v_{n} \theta\right) \in \operatorname{core} \rho$, and since core $\rho \in V_{u},\left(u\left(v_{1}, \ldots, v_{n}\right)\right) \theta \in E(S)$. Thus $S \in \mathcal{V}_{\mathbf{u}} * \mathcal{V}_{\mathbf{v}}$

We shall say that an inverse semigroup $S$ is an extension of an inverse semigroup $A$ by an inverse semigroup $B$ if there is a congruence, $\rho$, on $S$ such that $A \cong$ core $\rho$ and $B \cong S / \rho$. Thus we have just seen that the verbal product of varieties of inverse semigroups contains extensions. Now H. Newmann [12] defined a product of varieties of groups to be the collection of all extensions of groups in the first variety by groups of the second variety. She then showed that this product of varieties is equivalent to a verbal product of varieties of groups. This product defined by extensions does not translate readily into inverse semigroups, as J. Bales [1] in an example showed that such a product is not always a variety of inverse semigroups.

We now give a partial converse to Lemma 15 . We need the following lemma.

LEMMA 16. Let $\mathrm{w}$ be a total set of words and $S$ a semilattice of groups. $\mathrm{w}(S)$ is a fully invariant normal inverse subsemigroup of $S$.

Proof. By Lemma 12, all we need to show is that $w(S)$ is normal in $S$. Let $a b \in \mathrm{w}(S)$ and $d \in \mathrm{w}(S)$. Then $a b=u \alpha$ and $d=v \beta$ where $u, v \in \mathrm{w}$ and $\alpha$, 
$\beta \in \operatorname{Mor}\left(F I_{X}, S\right)$. Now choose $\phi \in \operatorname{Aut}\left(F I_{X}\right)$, induced by a permutation of $X$, such that $c(u) \cap c(v \phi)=\varnothing$. Define a morphism $\gamma: F I_{X} \rightarrow S$ by

$$
x_{i} \rightarrow \begin{cases}x_{i} \alpha, & \text { if } x_{i} \in c(u), \\ x_{i} \phi^{-1} \beta, & \text { if } x_{i} \in c(v \phi), \text { and } \\ b, & \text { otherwise. }\end{cases}
$$

Choose $x_{k} \in X$, not in $c(u) \cup c(v \phi)$. Now by Lemma 11 , $w$ is a fully invariant normal inverse subsemigroup of $F I_{X}$. Thus since $x_{k}^{-1} x_{k} \in E\left(F I_{X}\right) \subseteq \mathrm{w}$ and $v \phi \in$ $\mathbf{w} \phi \subseteq \mathbf{w}$, then $x_{k}^{-1}(v \phi) x_{k}$ and hence $u x_{k}^{-1}(v \phi) x_{k} \in \mathbf{w}$. Thus $\left(u x_{k}^{-1}(v \phi) x_{k}\right) \gamma \in \mathbf{w}(S)$ so that $\left((u \gamma) x_{k}^{-1} \gamma\right)\left((v \phi \gamma) x_{k} \gamma\right)=a b b^{-1} d b=a d b \in \mathbf{w}(S)$, since idempotents lie in the centre of a semilattice of groups. Thus $\mathrm{w}(S)$ is normal.

THEOREM 17. Let $\mathfrak{V}_{\mathrm{u}}$ and $\mathfrak{V}_{\mathrm{v}}$ be verbal varieties. Let $S$ be a semilattice of groups. $S \in \mathscr{V}_{u} * \mathscr{V}_{v}$ if and only if there is a congruence $\rho$ on $S$ such that core $\rho \in \mathscr{V}_{u}$ and $S / \rho \in \mathscr{V}_{v}$, that is $S$ is an extension of a member of $\mathscr{V}_{u}$ by a member of $\mathscr{V}_{v}$.

Proof. We have shown one half of the result already in Lemma 15, so now to show the other half, let $S \in \mathscr{V}_{\mathbf{u}} * \mathscr{V}_{\mathbf{v}}$. Without loss of generality, we assume that $\mathbf{u}$ and $\mathbf{v}$ are total. We shall show that $\rho_{\mathrm{v}(S)}$ meets the requirements of the theorem. Now by Lemma 9 , core $\rho_{\mathrm{v}(S)}=\mathrm{v}(S)$. Thus we must show that $\mathrm{v}(S) \in \mathfrak{T}_{\mathrm{u}}$. Let $u \in \mathbf{u}$ and $\theta \in \operatorname{Mor}\left(F I_{X}, \mathbf{v}(S)\right)$. Since the naming of elements in $X$ was arbitrary, we assume that $c(u)=\left\{x_{1}, \ldots, x_{n}\right\}$. Now for each $x_{i} \in c(u), x_{i} \theta \in \mathrm{v}(S)$, so we may pick $v_{i} \in \mathrm{v}$ and $\alpha_{i} \in \operatorname{Mor}\left(F I_{X}, S\right)$, with $x_{i} \theta=v_{i} \alpha_{i}$, and using the full invariance of $\mathrm{v}$, we may assume that $c\left(v_{i}\right) \cap c\left(v_{j}\right)=\varnothing$ for $i \neq j, i, j=1,2, \ldots, n$. Define $\beta: F I_{X} \rightarrow S$ by

$$
\beta: \begin{cases}x_{i} \rightarrow x_{i} \alpha_{i}, & \text { if } x_{i} \in c\left(v_{i}\right) \text { for } x_{i} \in \cup_{j=1}^{n} c\left(v_{j}\right), \\ x_{i} \rightarrow s, & \text { for some arbitrary } s \in S, \text { otherwise }\end{cases}
$$

Thus $v_{i} \beta=v_{i} \alpha_{i}$ for $i=1, \ldots, n$.

Now

$$
\begin{aligned}
u \theta & =u\left(x_{1}, \ldots, x_{n}\right) \theta \\
& =u\left(x_{1} \theta, \ldots, x_{n} \theta\right) \\
& =u\left(v_{1} \alpha_{1}, \ldots, v_{n} \alpha_{n}\right) \\
& =u\left(v_{1} \beta, \ldots, v_{n} \beta\right) \\
& =u\left(v_{1}, \ldots, v_{n}\right) \beta .
\end{aligned}
$$

But as $S \in \mathscr{V}_{\mathrm{u}} * \mathscr{V}_{\mathbf{v}}$ and $u\left(v_{1}, \ldots, v_{n}\right) \in \mathbf{u}(\mathbf{v})$, then $u\left(v_{1}, \ldots, v_{n}\right) \beta$, and hence $u \theta \in E(S)$. Thus, since $\mathbf{v}(S)$ is normal in $S, E(\mathbf{v}(S))=E(S)$ and so $\mathbf{v}(S) \in \mathfrak{V}_{u}$. 
It remains to show that $S / \rho_{\mathrm{v}(S)} \in \mathscr{V}_{\mathrm{v}}$. Define $\theta_{1} \in \operatorname{Mor}\left(F I_{X}, S\right)$ by $x_{i} \theta_{1}=s_{i}$ where $s_{i}$ is any element of $x_{i} \theta$. Then $\theta=\theta_{1}\left(\rho_{\mathrm{v}(S)}\right)$ and

$$
\begin{aligned}
v \theta & =v\left(\theta_{1}\left(\rho_{v(S)}\right)\right) \\
& =\left(v \theta_{1}\right)\left(\rho_{v(S)}\right) \\
& =\left(v v^{-1} \theta_{1}\right)\left(\rho_{v(S)}\right), \quad \text { by definition of } \rho_{v(S)} \\
& =\left(v v^{-1}\right)\left(\theta_{1}\left(\rho_{v(S)}\right)\right) \\
& =\left(v v^{-1}\right) \theta \\
& =(v \theta)(v \theta)^{-1} .
\end{aligned}
$$

Thus $v \theta \in E\left(S / \rho_{\mathrm{v}(S)}\right)$ and hence $S / \rho_{\mathrm{v}(S)} \in \mathscr{V}_{\mathrm{v}}$.

\section{The lattice of verbal varieties}

After the initial remarks concerning verbal varieties made in Section 1, we now wish to examine the lattice of verbal varieties, denoted $\operatorname{eV}(9 S)$ in a little more detail. First observe the following well-known result, as quoted by Bales [1].

LEMMA 18. Let $S$ be an inverse semigroup. Then $S$ is a group if and only if $S$ satisfies an identity $(u, v)$ where $c(u) \neq c(v)$.

We can thus observe that verbal varieties of inverse semigroups never coincide with varieties of groups. However by investigating the structure of the lattice of verbal varieties, we strengthen this result to see that a verbal variety never consists solely of semi-lattices of groups.

First we need definitions of meet and join in $\mathcal{L V}(9 S)$. The meet of two verbal varieties is the largest verbal variety contained in both of them; the join of two verbal varieties is the smallest verbal variety containing both of the varieties. It is easy to see that the meet of two verbal varieties $\mathcal{V}_{\mathbf{u}}$ and $\mathcal{V}_{\mathbf{v}}$ in $\mathcal{L}(9 \delta)$ coincides with the meet in $\mathcal{L}(9 \delta)$, the lattice of varieties of inverse semigroups. Thus we have:

LeMma 19. Let $\mathscr{V}_{u}$ and $\mathscr{V}_{v}$ be verbal varieties. In $\mathcal{L} \mathcal{V}_{(}(\S) \mathscr{V}_{u} \wedge \mathscr{V}_{\mathbf{v}}=\mathscr{V}_{u \cup v}$, the variety defined by the union of the generating set of words, $\mathbf{u}$ and $\mathbf{v}$.

In $\mathcal{L}(9 \delta)$, the join of $\mathcal{V}_{u}$ and $\mathcal{V}_{v}$ in the variety defined by $\rho_{u}^{*} \cap \rho_{v}^{*}$, the intersection of the fully invariant congruences generated by $\left\{\left(u, u u^{-1}\right) \mid u \in \mathbf{u}\right\}$ 
and $\left\{\left(v, v v^{-1}\right) \mid v \in \mathbf{v}\right\}$ respectively. Now consider all pairs $\left(w, w w^{-1}\right) \in \rho_{u}^{*} \cap \rho_{v}^{*}$ and denote this set by $w$. Then $\mathscr{V}_{w}$ is the smallest verbal variety that contains both $\mathscr{V}_{u}$ and $\mathscr{V}_{v}$. Now consider the verbal variety $\mathcal{V}_{T(u) \cap T(v)} . \mathcal{V}_{u}, \mathcal{V}_{v} \subseteq \mathcal{V}_{T(\mathrm{u}) \cap T(\mathrm{v})}$, so that $\mathscr{V}_{w}=\mathscr{V}_{\mathrm{u}} \vee \mathscr{V}_{v} \subseteq \mathcal{V}_{T(u) \cap T(v)}$. However if $w \in \mathrm{w}$ then $\left(w, w w^{-1}\right) \in \rho_{\mathrm{u}}^{*} \cap \rho_{\mathrm{v}}^{*}$, so $w \in$ $T(\mathbf{u}) \cap T(\mathrm{v})$. Thus $\mathbf{w} \subseteq T(\mathrm{u}) \cap T(\mathrm{v})$, so $\mathscr{V}_{T(\mathrm{u}) \cap T(\mathrm{v})}=\mathscr{V}_{\mathrm{w}}$. Thus we have shown:

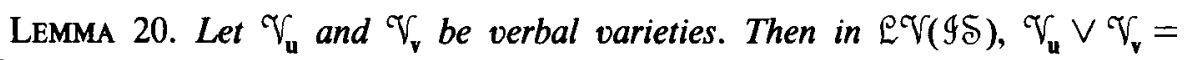
$\mathcal{V}_{T(\mathrm{u}) \cap T(\mathrm{v})}$.

We now consider where the verbal product sits in the lattice. In the case of groups, because of the product's alternative characterization in terms of extensions it is trivial to see that if $\mathcal{Q}$ and $\mathcal{V}$ are varieties of groups, then the verbal product contains both varieties. Thus if, $\mathcal{Q} * \mathfrak{V}$ denotes the verbal product of $\mathscr{U}$ and $\mathfrak{V}$ in the lattice of varieties of groups $\mathcal{L}(\mathcal{G})$, we have

LEMma 21. Let $\mathscr{Q}, \mathfrak{V}$ be varieties of groups. Then in $\mathscr{L}(\mathcal{G}), \mathscr{U} \vee \mathfrak{T} \leqslant$ $(\mathscr{Q} * \mathfrak{V}) \wedge(\mathfrak{V} * \mathscr{U})$

This result holds for $\mathcal{E} \mathcal{V}(9 \mathcal{S})$ as well.

LEMMA 22. Let $\mathcal{V}_{u}, \mathcal{V}_{v}$ be verbal varieties of inverse semigroups. Then in $\mathrm{E} \mathcal{T}(9 \delta)$,

$$
\mathscr{V}_{u} \vee \mathscr{V}_{v} \leqslant\left(\mathscr{V}_{u} * \mathscr{V}_{v}\right) \wedge\left(\mathscr{V}_{v} * \mathscr{V}_{u}\right)
$$

Proof. Let $w$ be a law of $\mathfrak{T}_{u} * \mathfrak{V}_{v}$; hence $w=u\left(v_{1}, \ldots, v_{n}\right)$ where $u$ is a law of $\mathcal{V}_{\mathrm{u}}$ and each $v_{i}$ is a law of $\mathcal{V}_{\mathrm{v}}$. Now consider the map $\theta \in \operatorname{Mor}\left(F I_{X}, F I_{X}\right)$ generated by

$$
\theta: \begin{cases}x_{i} \rightarrow v_{i}, & \text { if } x_{i} \in c(u), \text { and } \\ x_{i} \rightarrow x_{i}, & \text { otherwise. }\end{cases}
$$

Then $u \theta=w$. However $u$ is a law of $\mathscr{V}_{u}$ and the laws of $\mathscr{V}_{u}$ form a fully invariant subgroup of $F I_{X}$, so that $w$ is also a law of $\mathscr{V}_{u}$. Thus $\mathscr{V}_{u} \leqslant \mathscr{V}_{u} * \mathscr{V}_{v}$. Now if $S \in \mathscr{V}_{v}$, then for any $\theta \in \operatorname{Mor}\left(F I_{X}, S\right), v$ a law of $\mathcal{V}_{v}, v \theta \in E(S)$, so certainly $u\left(v_{1}, \ldots, v_{n}\right) \theta \in E(S)$, so $S \in \mathcal{V}_{u} * \mathcal{V}_{v}$. Thus $\mathscr{V}_{v} \leqslant \mathscr{V}_{u} * \mathscr{V}_{v}$. Hence $\mathscr{V}_{u} \vee \mathscr{V}_{v} \leqslant \mathscr{V}_{u}$ $* \mathscr{V}_{v}$ and by symmetry, $\mathscr{V}_{u} \vee \mathscr{V}_{v} \leqslant\left(\mathscr{V}_{u} * \mathscr{V}_{v}\right) \wedge\left(\mathscr{V}_{v} * \mathscr{V}_{u}\right)$

This inequality cannot be strengthened, as we can see in the following example. 
EXAmple 23. Let $C_{2}$ be the two element group, and let $\theta: C_{2} \rightarrow \operatorname{Aut}\left(C_{2} \times C_{2}\right)$ be given by

$$
\boldsymbol{\theta}:\left\{\begin{array}{l}
1 \rightarrow 1_{C_{2} \times C_{2}}, \\
a \rightarrow \boldsymbol{\alpha}: C_{2} \times C_{2} \rightarrow C_{2} \times C_{2},
\end{array}\right.
$$

where $\alpha$ is given by $(1, a) \alpha=(a, 1),(a, 1) \alpha=(1, a),(a, a) \alpha=(a, a)$ and $(1,1) \alpha$ $=(1,1)$.

Then we may form the semi-direct product of groups $C_{2}$ and $C_{2} \times C_{2}$ under $\theta$. This is the dihedral group, $D_{4}$. Now $C_{2}$ and $C_{2} \times C_{2}$ are both members of $\mathscr{V}_{\left\{x^{2}\right\}}$. However $D_{4}$, with multiplication,

$$
\left(x_{1}, y_{1}\right)\left(x_{2}, y_{2}\right)=\left(x_{1} x_{2},\left(y_{1}\left(x_{2} \theta\right)\right) y_{2}\right),
$$

is not a member of $\mathcal{V}_{\left\{x^{2}\right\}}$, since

$$
(a,(1, a))(a,(1, a))=(1,(a, a)) .
$$

Now $D_{4}$ is an extension of $C_{2} \times C_{2}$ by $C_{2}$ and so by Lemma $15, D_{4}$ is an element of $\widetilde{V}_{\left\{x^{2}\right\}} * \widetilde{V}_{\left\{x^{2}\right\}}$, which thus strictly includes $\widetilde{V}_{\left\{x^{2}\right\}} \vee \widetilde{V}_{\left\{x^{2}\right\}}=\widetilde{V}_{\left\{x^{2}\right\}}$.

We now wish to examine the atoms of $\operatorname{eV}(9 \delta)$ and as a consequence of this examination, show that no verbal variety consists solely of semilattices of groups.

Let $B_{2}$ denote the five element combinatorial Brandt semigroup; this inverse semigroup may be derived in various different ways.

(i) $B_{2}$ is isomorphic to the inverse subsemigroup of $I_{X}$, the symmetric inverse semigroup on $X$, generated by the map $\left(x_{1} \rightarrow x_{2}\right)$.

(ii) $B_{2} \cong M^{0}(1 ; I, I ; \Delta)$ where $|I|=2$.

(iii) $B_{2} \cong F I_{\{x\}} / \rho$ where $\rho$ is the fully invariant congruence on $F I_{\{x\}}$ generated by $\left(\left(x^{2} x^{-1}\right),\left(x^{2} x^{-1}\right)\left(x^{2} x^{-1}\right)^{-1}\right)$.

(iv) $B_{2}$ and $F I_{X / \rho}$, where $\rho$ is the fully invariant congruence generated by $\left(\left(x_{1} x_{2} x_{1}^{-1}\right),\left(x_{1} x_{2} x_{1}^{-1}\right)\left(x_{1} x_{2} x_{1}^{-1}\right)^{-1}\right)$ generate the same variety. See Reilly [13] and Kleiman [7].

THEOREM 24. Let $\mathrm{w}$ be a set of words in $F I_{X}$. If $\mathfrak{V}_{w} \neq S \mathcal{S}$, then $B_{2} \in \mathfrak{V}_{w}$.

Proof. Suppose $B_{2} \notin \mathfrak{V}_{w}$. Now $B_{2}$ has only two elements that are not idempotents. Let $x$ be a non-idempotent element of $B_{2}$. Thus there exist $w \in w$ and $\theta \in \operatorname{Mor}\left(F I_{X}, B_{2}\right)$ such that $w \theta=x$. Without loss of generality we may assume that $c(w)=\left\{x_{1}, \ldots, x_{n}\right\}$. Now define $\alpha \in \operatorname{End}\left(F I_{X}\right)$ by, for $i=1, \ldots, n, x_{i} \alpha=x_{1}$, $x_{1}^{-1}, x_{1} x_{1}^{-1}$ or $x_{1}^{-1} x_{1}$ according as to whether $x_{i} \theta=x, x^{-1}, x x^{-1}$ or $x^{-1} x$, and for $i>n, x_{i} \alpha=x_{2}$. (Clearly $x_{i} \theta \neq 0$ for $i=1, \ldots, n$.) Now $x$ in $B_{2}$ and $x_{1}$ in $F I_{X}$ share the property that they are the product of a string $a_{1}, \ldots, a_{m}$ if and only if 
the string can be expanded to $x x^{-1} x x^{-1} x \ldots x^{-1} x$ (or $x_{1} x_{1}^{-1} x_{1} x_{1}^{-1} \ldots x_{1}^{-1} x_{1}$ respectively), so that since $w \theta=x, w \alpha=x_{1}$. Thus, since $T(w)$ is a fully invariant inverse subsemigroup of $F I_{X}, x_{1} \in T(w)$ and so $T(w)=F I_{X}$. Thus $\mathcal{V}_{w}=\mathcal{S E}$.

COROllary 1. $\mathbb{V}_{\left\{x_{1} x_{2} x_{1}^{-1}\right\}}$ is the only atom of $\mathcal{L} \mathcal{V}(9 S)$.

Proof. By the fourth formulation of $B_{2}$, above, the variety defined by $B_{2}$ in $9 \mathcal{S}$ is the verbal variety $\mathcal{V}_{\left\{x_{1} x_{2} x_{1}^{-1}\right\}}$ and so by the theorem $\widetilde{V}_{\left\{x_{1} x_{2} x_{1}^{-1}\right\}} \subseteq \mathcal{V}_{w}$, for any non-trivial verbal variety.

COROLlaRY 2. No non-trivial verbal variety consists solely of semilattices of groups.

Proof. $B_{2}$ is not a semilattice of groups.

\section{References}

[1] J. Bales, 'On product varieties of inverse semigroups' (Algebra Paper 31, Department of Mathematics, Monash University, 1978).

[2] G. Birkhoff, 'On the structures of abstract algebras', Proc. Cambridge Philos. Soc. 31 (1935), 433-454.

[3] A. H. Clifford and Preston, G. B., The algebraic theory of semigroups, Vol. I. (Amer. Math. Soc. Math. Surveys 7, Providence, R.I., 1961).

[4] G. G. Djadchenko, 'On identities in monogenic inverse semigroups', Algebra and Theory of Numbers, Kabardino-Balkarsk Gos. Univ. Učen. Zap. (Nalcik), 2 (1977), 57-77, (Russian).

[5] D. Green, 'The lattice of congruences on an inverse semigroups', Pacific J. Math. 57 (1975), $141-152$.

[6] J. M. Howie, An introduction to semigroup theory, (Academic Press, London, 1976).

[7] E. I. Kleinmann, 'On basis of identities of Brandt semigroups', Semigroup Forum 13 (1977), 209-218.

[8] A. G. Kurosh, The theory of groups, 2nd ed. 1953, (Translation in English by K. A. Hirsch, Chelsea, 1956).

[9] A. I. Mal'cev, 'Multiplication of classes of algebraic systems', Siberian Math. J. 8 (1967), $254-267$.

[10] S. Moran, 'Associative operations on groups I', Proc. London Math. Soc. 6 (1956), 581-596.

[11] B. H. Neumann, 'Identical relations in groups. I', Math. Ann. 114 (1937), 506-525.

[12] H. Neumann, Varieties of groups, (Ergebnisse der Mathematik und ihre Grenzgebiete, 37, Springer-Verlag, Berlin, 1967).

[13] N. R. Reilly, Varieties of inverse semigroups, Proceedings of the Conference on Semigroups in Honour of Alfred H. Clifford, pp. 85-93, Tulane University, New Orleans 1979.

\section{Department of Mathematics}

Monash University

Clayton, Victoria

Australia 3168 